\title{
Multiple-QR-Decomposition Assisted Group Detection for Reduced-Complexity-and-Latency MIMO-OFDM Receivers
}

\author{
Ryota KIMURA $^{\dagger \ddagger}$, Ryuhei FUNADA ${ }^{\dagger}$, Hiroshi HARADA ${ }^{\dagger}$, and Shigeru SHIMAMOTO H $^{\ddagger}$ \\ ${ }^{\dagger}$ National Institute of Information and Communications Technology \\ YRP 1 Bldg., 3-4 Hikarino-Oka, Yokosuka-Shi, Kanagawa, 239-0847 Japan \\ E-mail: \{r.kimura, funada, harada\}@nict.go.jp \\ ${ }^{\ddagger}$ Graduate School of Global Information and Telecommunication Studies, Waseda University \\ Waseda Univ. 29-7 Bldg., 1-3-10 Nishi-Waseda, Shinjuku-Ku, Tokyo, 169-0051 Japan \\ E-mail: kimura@sl.giti.waseda.ac.jp, shima@waseda.jp
}

\begin{abstract}
We propose a new multiple-QR-decomposition assisted group detection (Multi-QRD-GD) algorithm for the development of reduced-complexity-and-latency multi-input multi-output (MIMO) orthogonal frequency division multiplexing (OFDM) receivers. In addition, we investigate an effect of adaptive grouping (AG) based on received signal-to-noise power ratio for Multi-QRD-GD. In this paper, we describe the functions of the Multi-QRD-GD algorithm and AG scheme. Then, we compare bit error rate (BER) performances of the proposed Multi-QRDGD and conventional detection algorithms by computer simulations. In addition, we compare the computational complexities of the algorithms.
\end{abstract}

\section{INTRODUCTION}

Multi-input multi-output (MIMO) techniques, especially space division multiplexing (SDM) [1], are promising approaches to realize high spectrum efficiency and high data-rate in wireless communications. The MIMO techniques employ multiple antennas both in a transmitter and receiver sides. The transmitter simultaneously transmits different signals in a common frequency band from the multiple antennas. Then, the receiver detects and decomposes those signals with using some signal detection algorithms. The spectrum efficiency proportionally increases to the number of transmit antennas in SDM. In addition, orthogonal frequency division multiplexing (OFDM) [2] is also useful for the high spectrum efficient wireless systems. OFDM is a multi-carrier based transmission, and robust against intersymbol interference (ISI) and frequency selective channel. Recently, the combined MIMO-OFDM techniques have been evaluated in the world [3], [4].

Signal detection algorithms are very important part in SDM. The transmission performance of SDM depends on the algorithms. Unfortunately, there is a trade-off between the performance and the computational complexity of the algorithms. The simplest algorithm is spatial filtering [1] based on zero-forcing (ZF) or minimum mean squared error (MMSE) criteria. Maximum likelihood detection (MLD) [5] provides the best performance, though it requires the highest complexity. The MLD's complexity increases proportionally to a constellation size of a modulation scheme and exponentially to the number of transmit antennas. In recent studies, many proposals [6]-[13] have tried to reduce the MLD's complexity while nearly keeping its performance.

We consider an application of MIMO-OFDM to mobile communication systems supporting high-mobility vehicle users. Sequentiallyupdated channel estimation (SUCE) [14] is an useful scheme to reduce channel estimation errors due to a fast time-varying fading channel. However, a latency time of the receiver's processing induces irreducible estimation errors in such a channel [14]. To avoid the errors, we focus on reducing the latency time as well as reducing the complexity in the MLD's signal processing. Group detection (GD) [9], [10] has an advantage in term of the latency time, since it carries out multiple local detectors in parallel. In this paper, we apply GD to the MIMO-OFDM receivers and propose new multipleQR-decomposition assisted group detection (Multi-QRD-GD). MultiQRD is performed as a sub-space projector to separate transmitted symbols into multiple sub-groups in Multi-QRD-GD, though ZF or MMSE is performed in the conventional GD. Moreover, the M-algorithm is performed in the local detectors to generate loglikelihood ratio (LLR) values and reduce the detectors' complexity. With a help of Multi-QRD-GD, we can directly concatenate the subspace projector and the local detectors. Moreover, we can reduce the latency time because Multi-QRD-GD never uses any iterative processing. In addition, we investigate on adaptive grouping (AG) schemes based on received signal-to-noise power ratio (SNR) values to improve the performance of the local detectors.

The rest of this paper is organized as follows. In Sect. II, we describe an MIMO-OFDM system and its signal model. In Sect. III, we present the functions of our proposed Multi-QRD-GD and AG. We evaluate bit error rate (BER) performances of the proposed algorithm by computer simulations in Sect. IV. Moreover, we compare the complexities of the proposed and conventional detection algorithms in Sect. V. In Sect. VI, we conclude this paper and refer to our future works.

\section{MIMO-OFDM SYSTEM MODEL}

We use a MIMO-OFDM system model as illustrated in Fig. 1 in this paper. We assume that the number of transmitter antennas, $L$, and that of receiver anntenas, $M$, are same. A transmitter consists of ordinary MIMO-OFDM blocks, such as a forward error correction (FEC) coder, interleavers, serial-to-parallel (S/P) converters, subcarrier modulators, inverse fast Fourier transform (IFFT) circuits, and GI adders. We define $s_{l}(t, k)$ as a frequency-domain symbol transmitted from the $l$-th antenna on the $k$-th sub-carrier at time $t$. Similarly, we define a transmitted symbol vector $\boldsymbol{s}(t, k)=$ $\left[s_{1}(t, k) \cdots s_{L}(t, k)\right]^{T}$. A receiver consists of a Multi-QRD-GD block in addition to GI removers, FFT circuits, channel estimator, parallel-to-serial (P/S) converters, de-interleavers, and FEC decoder. The symbols from the transmitter arrive at the receiver after passing through a MIMO channel. The symbol vector of the FFT output, $\boldsymbol{r}(t, k)$, can be expressed as follows:

$$
\begin{aligned}
\boldsymbol{r}(t, k) & =\left[\begin{array}{lll}
r_{1}(t, k) & \cdots & r_{M}(t, k)
\end{array}\right]^{T} \\
& =\boldsymbol{H}(t, k) \boldsymbol{s}(t, k)+\boldsymbol{n}(t, k), \\
\boldsymbol{H}(t, k) & =\left[\begin{array}{lll}
\boldsymbol{h}_{1}(t, k) & \cdots & \boldsymbol{h}_{L}(t, k)
\end{array}\right] \\
\boldsymbol{h}_{l}(t, k) & =\left[\begin{array}{lll}
h_{1, l}(t, k) & \cdots & h_{M, l}(t, k)
\end{array}\right]^{T}, \\
\boldsymbol{n}(t, k) & =\left[\begin{array}{lll}
n_{1}(t, k) & \cdots & n_{M}(t, k)
\end{array}\right]^{T},
\end{aligned}
$$




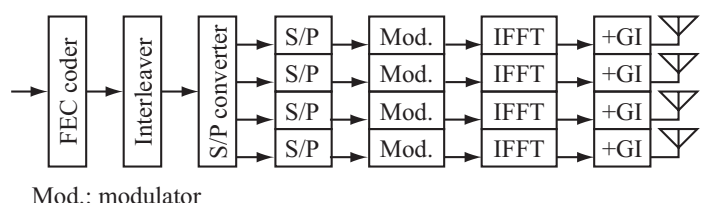

(a) Transmitter

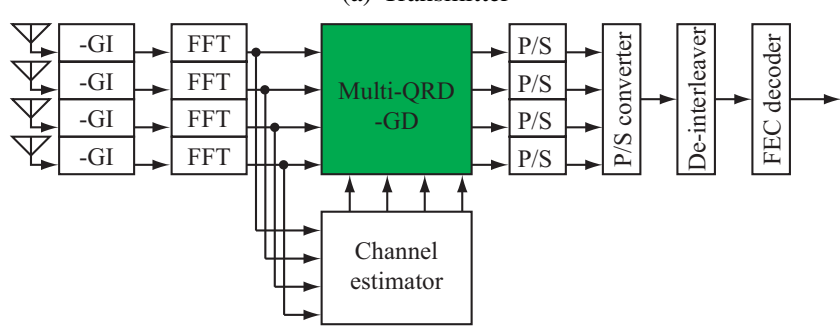

(b) Receiver

Fig. 1. MIMO-OFDM system model.

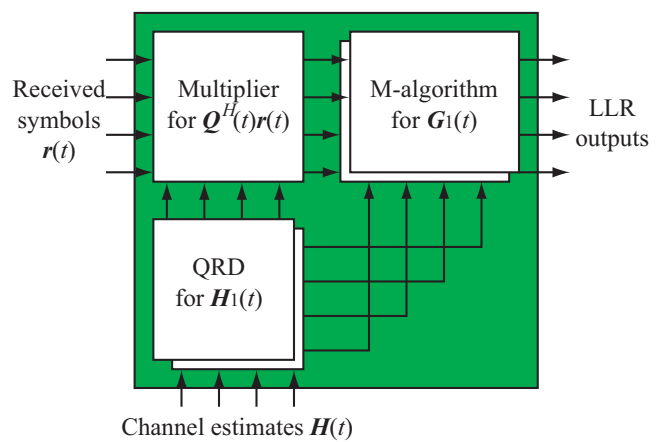

Fig. 2. Configuration of Multi-QRD-GD.

where $r_{m}(t, k)$ is the symbol received by the $m$-th receive antenna on the $k$-th sub-carrier at time $t, h_{m, l}(t, k)$ is the $k$-th sub-carrier's channel coefficient between the $l$-th transmitter and the $m$-th receiver antennas, and $n_{m}(t, k)$ is an additive white Gaussian noise (AWGN) component with zero mean and $\sigma^{2}$ variance. In this paper, we assume that the channel coefficients are spatially identical and independent each other.

\section{Multiple-QR-Decomposition Assisted Group DETECTION}

Figure 2 illustrates the configuration of our proposed Multi-QRDGD block. The conventional GD [9] consists of a space projector by ZF or MMSE and local detectors by Full-MLD. In contrast, our proposed Multi-QRD-GD consists of two parts; a space projector assisted by Multi-QRD, and local detectors with the M-algorithm.

\section{A. Principle}

First, Multi-QRD-GD generates arbitrary number $G_{r}$ of subgroups, each of which includes a set of the transmitted symbols. Basically, it can generate sub-groups having arbitrary size and elements. However, we aim at reducing both the complexity and latency time in the receiver. Then, we apply two conditions, (i)the sub-groups' sizes are same each other, and (ii)the sub-groups are not overlapped each other. In following, we introduce a case that $L$ and $M$ are equal to four, and $G_{r}$ is two.

For the simplification, we abbreviate the indices $t$ and $k$. We redefine $\boldsymbol{H}_{1}=\boldsymbol{H}$. The matrix $\boldsymbol{H}_{1}$ is decomposed into an unitary matrix, $\boldsymbol{Q}_{1}$, and upper triangular one, $\boldsymbol{R}_{1}$, i.e. $\boldsymbol{H}_{1}=\boldsymbol{Q}_{1} \boldsymbol{R}_{1}$, where

$$
\begin{aligned}
\boldsymbol{Q}_{1}= & {\left[\begin{array}{ccc}
q_{1,1,1} & \cdots & q_{1,4,1} \\
\vdots & \ddots & \vdots \\
q_{4,1,1} & \cdots & q_{4,4,1}
\end{array}\right], } \\
\boldsymbol{R}_{1}= & {\left[\begin{array}{cccc}
\xi_{1,1,1} & \cdots & \cdots & \xi_{1,4,1} \\
0 & \xi_{2,2,1} & \ddots & \vdots \\
\vdots & \ddots & \ddots & \vdots \\
0 & \cdots & 0 & \xi_{4,4,1}
\end{array}\right] . }
\end{aligned}
$$

After that, we obtain a vector $\boldsymbol{z}_{1}$ by multiplying $\boldsymbol{Q}_{1}^{H}$ with $\boldsymbol{r}$ :

$$
\begin{aligned}
\boldsymbol{z}_{1} & =\boldsymbol{Q}_{1}^{H} \boldsymbol{r} \\
& =\boldsymbol{R}_{1} \boldsymbol{s}+\tilde{\boldsymbol{n}}_{1},
\end{aligned}
$$

where $\tilde{\boldsymbol{n}}_{1}=\boldsymbol{Q}_{1}^{H} \boldsymbol{n}$. The operator $\boldsymbol{A}^{H}$ indicates conjugate transposition of a matrix $\boldsymbol{A}$. The last half of $\boldsymbol{z}_{1},\left[\begin{array}{lll}z_{3,1} & \cdots & z_{4,1}\end{array}\right]^{T}$, can be written as follows:

$$
\left[\begin{array}{l}
z_{3,1} \\
z_{4,1}
\end{array}\right]=\left[\begin{array}{cc}
\xi_{3,3,1} & \xi_{3,4,1} \\
0 & \xi_{4,4,1}
\end{array}\right]\left[\begin{array}{l}
s_{3} \\
s_{4}
\end{array}\right]+\left[\begin{array}{l}
\tilde{n}_{3,1} \\
\tilde{n}_{4,1}
\end{array}\right]
$$

Equation (8) indicates that we obtain a sub-group including only $s_{3}$ and $s_{4}$. At the same time, another QRD generates matrices $\boldsymbol{Q}_{2}$ and $\boldsymbol{R}_{2}$ from a column-permutated channel matrix $\boldsymbol{H}_{2}=\left[\begin{array}{llll}\boldsymbol{h}_{3} & \boldsymbol{h}_{4} & \boldsymbol{h}_{1} & \boldsymbol{h}_{2}\end{array}\right]$. With the same manner by (7), we obtain the other sub-group including only $s_{1}$ and $s_{2}$ as follow:

$$
\left[\begin{array}{l}
z_{3,2} \\
z_{4,2}
\end{array}\right]=\left[\begin{array}{cc}
\xi_{3,3,2} & \xi_{3,4,2} \\
0 & \xi_{4,4,2}
\end{array}\right]\left[\begin{array}{l}
s_{1} \\
s_{2}
\end{array}\right]+\left[\begin{array}{l}
\tilde{n}_{3,2} \\
\tilde{n}_{4,2}
\end{array}\right] .
$$

We have separately explained the behavior of each sub-space projector by twice matrix multiplications of $\boldsymbol{Q}_{1}^{H} \boldsymbol{r}$ and $\boldsymbol{Q}_{2}^{H} \boldsymbol{r}$. However, we note that we can implement the sub-space projector into single matrix multiplication with taking account of the structures of $\boldsymbol{Q}_{1}$ and $Q_{2}$.

Each of output of the sub-space projector is fed into different local detector. We can directly and smoothly concatenate the subspace projector and the reduced-complexity local detectors employing QRD, such as sphere decoder [7] or M-algorithm [11]-[13], because the sub-space projector itself consists of QRDs. We apply the Malgorithm with a parameter for the number of surviving paths, $S_{1}$, to the detector to reduce the amount of the computation and generate log-likelihood ratio (LLR) values. Each detector first computes metrics based on squared Euclidean distances with the M-algorithm, and then generates LLR of coded bits. LLR of the $i$-th bit in the symbol $s_{l}, b_{l, i}$, is generated by the following computation:

$$
\begin{aligned}
b_{l, i} & =\sqrt{\mu_{l, i, 0, \min }}-\sqrt{\mu_{l, i, 1, \min }}, \\
\mu_{l, i, a, \min } & =\min \left\{\mu_{l, i, a}\right\},
\end{aligned}
$$

where $\mu_{l, i, a}$ is the metric computed by a replica symbol $\hat{s}_{l}$, the $i$ th bit of which indicates " $a \in\{0,1\}$. " LLRs are fed into the P/S converters, de-interleaver, and FEC decoder. Then, the FEC decoder recovers the transmitted bit stream.

\section{B. Adaptive Grouping Scheme}

We have introduced a simple grouping scheme without any consideration in the above sub-section. In this sub-section, we apply AG schemes to Multi-QRD-GD to improve the performance of the local detectors. 
Our proposed AG scheme is based on a ranking of SNR value for the symbols. First, a SNR value for $s_{l}, \gamma_{l}$, is measured by the following computation:

$$
\gamma_{l}=\left\|\boldsymbol{h}_{l}\right\|^{2}
$$

The operator $\|\boldsymbol{a}\|$ indicates a Euclidean norm of a vector $\boldsymbol{a}$. We note that $\gamma_{l}$ is a relative SNR value among $s_{l}$. We prepare a parameter $\operatorname{rank}(v)$ for $v=1, \cdots, L$. Then, we rank the indices $l$ based on a descending order of $\gamma_{l}$, e.g. $\operatorname{rank}(1)=\arg _{l} \max \left\{\gamma_{l}\right\}$ and $\operatorname{rank}(L)=\arg _{l} \min \left\{\gamma_{l}\right\}$. We decide the elements in each subgroup in according to this ranking. The appropriate grouping scheme helps the local detectors to generate more reliable LLR values, because the SNR values for the symbols are independent each other and the performance of the detectors depends on partial grouping and ordering of the symbols.

We investigate four types of the AG schemes, AG1 to AG4, in case that $L=4$ and $G_{r}=2$. Each AG scheme generates the columnpermutated channel matrices $\boldsymbol{H}_{1}$ and $\boldsymbol{H}_{2}$ as follows:

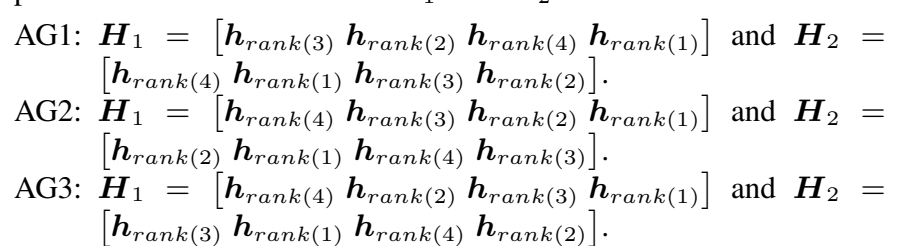
AG4: In this case, no consideration on the ranking is employed for the grouping, i.e. fixed grouping. $\boldsymbol{H}_{1}=\left[\boldsymbol{h}_{1} \boldsymbol{h}_{2} \boldsymbol{h}_{3} \boldsymbol{h}_{4}\right]$ and $\boldsymbol{H}_{2}=\left[\begin{array}{llll}\boldsymbol{h}_{3} & \boldsymbol{h}_{4} & \boldsymbol{h}_{1} & \boldsymbol{h}_{2}\end{array}\right]$.

\section{Consideration of Receiver's signal processing and Latency Time}

In this sub-section, we refer to a relationship between the receiver's signal processing and the latency time. Figure 3 shows the differentia between QR-decomposition and M-algorithm assisted MLD (QRMMLD) [13] and Multi-QRD-GD when $L=M=4$ and quadrature phase shift keying (QPSK) is applied. QRM-MLD requires a serial four-stage M-algorithm to detect the transmitted symbols. Such a serial multi-stage algorithm increases the latency time. In addition, propagation errors accumulate in the process of the algorithm. On the other hand, Multi-QRD-GD divides the MIMO symbols into a certain number of the sub-groups, e.g. 2 sub-groups in this paper. Accordingly, it carries out the local detectors in parallel, and thus reduces the latency time in the signal processing. We note that there is a trade-off between the latency time and diversity gain of GD. These factors depend on the number of elements in each sub-group. When the number of elements in each sub-group increases, it is difficult to reduce the latency time even though the local detectors are performed in parallel.

We consider that a real-time processing receiver more improves an actual data throughput without a huge amount of memories than a packet-by-packet processing one, especially in high data-rate wireless communication systems. In addition, we have confirmed that the excessive latency time degrades the performance of the real-time processing receiver especially in a fast time-varying fading environment [14]. Multi-QRD-GD can avoid such degradation because it reduces not only the computational complexity but also the latency time.

\section{Computer Simulation Results}

We evaluate bit error rate (BER) performances of the proposed Multi-QRD-GD algorithm by computer simulations. Table I shows the major parameters used in the simulations. We evaluate the $4 \times 4$ MIMO-OFDM system, i.e. $L=M=4$. Total bandwidth is 96 $\mathrm{MHz}$ and the number of sub-carriers, $K$, is 768 . IFFT/FFT points,

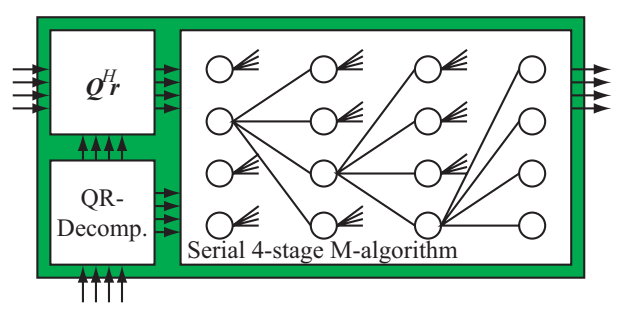

(a) QRM-MLD

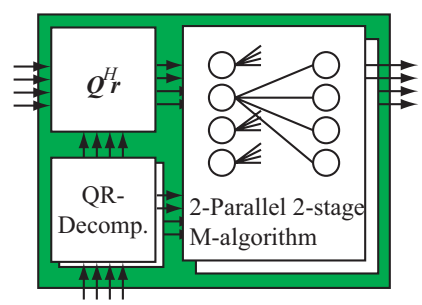

(b) Multi-QRD-GD

Fig. 3. Differentia between QRM-MLD and Multi-QRD-GD.

TABLE I

MAJOR PARAMETERS IN SIMULATION.

\begin{tabular}{l|l}
\hline Data rate & $307.2 \mathrm{Mbps}$ (QPSK) \\
& $614.4 \mathrm{Mbps}(16 \mathrm{Q} \mathrm{M})$ \\
\hline Number of antennas: $(L, M)$ & $(4,4)$ \\
\hline Total bandwidth & $96 \mathrm{MHz}$ \\
\hline Number of sub-carriers: $K$ & 768 \\
\hline IFFT/FFT points: $F$ & 1024 \\
\hline FEC scheme & Convolutional coding \\
& (rate $=1 / 2$, constraint $=7) /$ \\
& Soft decision Viterbi decoding \\
\hline Modulation & QPSK and 16QAM \\
\hline Effective symbol period & $8.0 \mu$ sec \\
\hline GI period & $2.0 \mu$ sec \\
\hline Number of pilot symbols & 2 \\
per packet: $P$ & \\
\hline Number of data symbols & 19 \\
per packet: $D$ & \\
\hline Synchronization & Perfect \\
\hline \hline Multi-path model & Exponentially decaying \\
\hline Path duration & $24-$ path Rayleigh fading \\
\hline Maximum delay & $62.5 \mathrm{nsec}$ \\
\hline RMS delay spread & $1.4375 \mu$ sec \\
\hline Maximum Doppler frequency: $f_{D}$ & $360 \mathrm{nsec}$ \\
\hline & $10 \mathrm{~Hz}(2 \mathrm{~km} / \mathrm{h}$ in $5 \mathrm{GHz})$ \\
\hline
\end{tabular}

$F$, are 1024. We apply convolutional coding and soft decision Viterbi decoding to an FEC technique, and quadrature phase shift keying (QPSK) and 16 quadrature amplitude modulation (QAM) to the subcarrier modulation. The effective symbol and GI periods in OFDM symbol are 8.0 and $2.0 \mu \mathrm{sec}$, respectively. Each transmitter antenna transmits a packet consisting of 2 pilot and 19 data OFDM symbols. We apply carrier interferometry based channel estimation [15] at the receiver. We assume that frequency and timing synchronizations are performed perfectly. The multi-path model used in the simulations is an exponentially decaying 24-path Rayleigh fading with a root mean square (RMS) delay spread of $360 \mathrm{nsec}$. A maximum Doppler frequency, $f_{D}$, is fixed to $10 \mathrm{~Hz}$. This value means that a user moves with his velocity of about $2 \mathrm{~km} / \mathrm{h}$ in a $5 \mathrm{GHz}$ wireless system.

Figure 4 shows the effect of our proposed AG schemes for the BER performances of Multi-QRD-GD. We confirm that AG1 and 


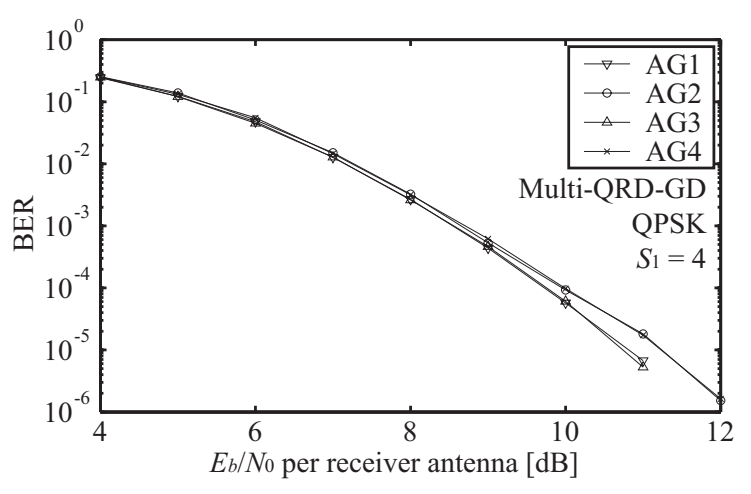

(a) QPSK

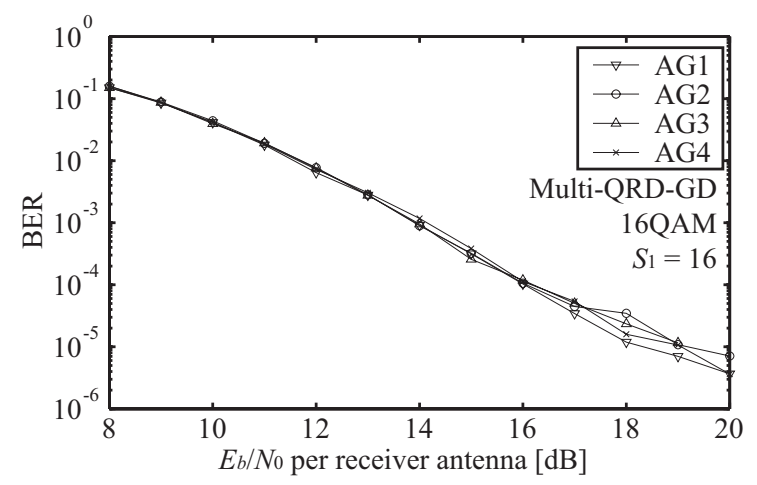

(b) 16QAM

Fig. 4. Effect of AG schemes for BER of Multi-QRD-GD.

AG3 improve the BER performances compared to AG2 and AG4 when QPSK is applied. This reason is that the combination of the symbols having the higher and lower SNR values effectively increases the reliability of the decision in the local detector. On the other hand, the performance difference among the AG schemes is reduced when 16QAM is applied. However, AG1 is slightly superior to the other AG schemes because of the same reason above. We apply AG1 to Multi-QRD-GD in following simulations.

Figure 5 shows the effect of the number of the surviving paths for each local detector, $S_{1}$. We set $S_{1}$ to $4,3,2$, and 1 when QPSK is applied, and set $S_{1}$ to $16,14,12$, and 8 when 16QAM is applied. When QPSK is applied, the local detectors require the enough large number of $S_{1}$ to achieve lower BER. Because QPSK's constellation size is basically small, the local detectors induce irreducible errors when $S_{1}$ is less than that size. On the other hand, when 16QAM is applied, the BER performance using $S_{1}=14$ is equivalent to that using $S_{1}=16$ down to BER $=10^{-4}$. This result means that the highlevel modulation is tolerant to the surviving path reduction because relatively a large number of candidates remain after the reduction. We employ $S_{1}=4$ for QPSK, and $S_{1}=16$ and 14 for 16QAM in following simulations.

Figure 6 compares the BER performances of Full-MLD, QRMMLD [13], and our proposed Multi-QRD-GD algorithms. In this simulation, Full-MLD also inputs LLR values to the FEC decoder. We set the numbers of the surviving paths for QRM-MLD, [ $\left.S_{1}, S_{2}, S_{3}\right]$, to $[4,4,4]$ and $[12,12,12]$ when QPSK and 16QAM are applied, respectively. When QPSK is applied, the BER performance of Multi-QRD-GD is worse than QRM-MLD. When QPSK, QRMMLD achieves its diversity gain. On the other hand, Multi-QRDGD reduces its diversity gain by generating the multiple sub-groups.

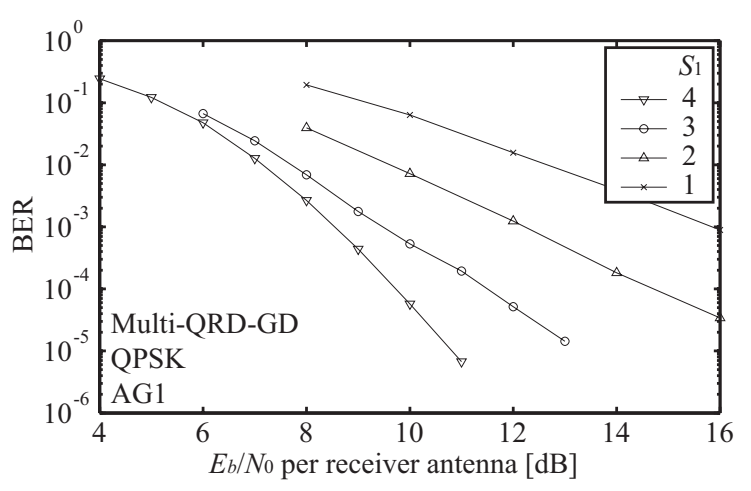

(a) QPSK

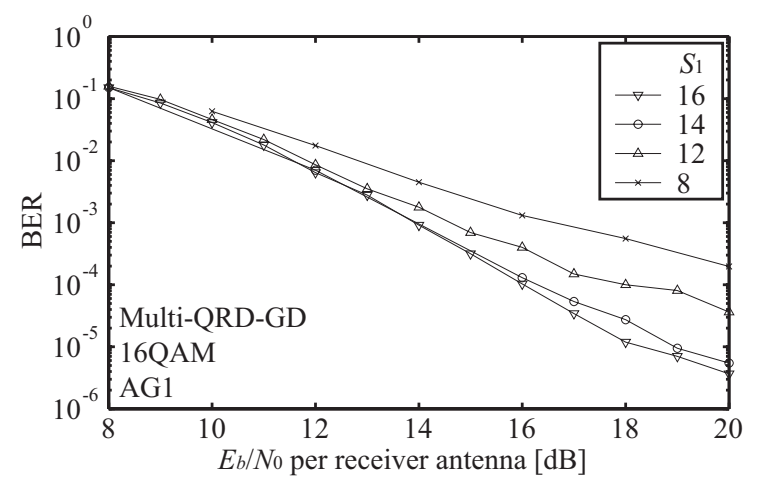

(b) 16QAM

Fig. 5. Effect of the number of surviving paths for BER of Multi-QRD-GD.

However, the loss of $E_{b} / N_{0}$ is approximately $0.5 \mathrm{~dB}$ at BER = $10^{-5}$. When 16QAM is applied, the BER performance of MultiQRD-GD achieves to that of QRM-MLD. QRM-MLD is sensitive to the propagation errors because all the stages are serially performed in single M-algorithm. This sensitivity degrades the BER performance especially when the number of the surviving paths is reduced. On the other hand, our Multi-QRD-GD efficiently avoids such propagation errors, despite the complexities of Multi-QRD-GD with both $S_{1}=16$ and 14 are lower than that of QRM-MLD.

\section{COMPLEXITY COMPARISON}

We discuss the complexities of the signal detection algorithms in this section. Table II shows the required numbers of the real multiplications in Full-MLD, QRM-MLD, and Multi-QRD-GD. We count those numbers under assumptions that a complex multiplication includes four real multiplications. In Table II, $S$ indicates the constellation size of modulation, e.g. $S=4$ and 16 for QPSK and 16QAM, respectively. We use the parameters shown in Table I to calculate the examples of the total number of the multiplications. We confirm that Multi-QRD-GD requires less complexity than QRM-MLD under the conditions of those parameters. When QPSK, Multi-QRD-GD reduces approximately 91 and 8 percents of the multiplications compared to MLD and QRM-MLD, respectively. When 16QAM, Multi-QRD-GD employing $S_{1}=16$ and 14 reduce approximately 8 and 18 percents of those compared to QRM-MLD, respectively. From these facts and the above evaluations, Multi-QRD-GD is an effective algorithm to achieve all the good BER performance, complexity, and latency in the receivers at once. 
The 17th Annual IEEE International Symposium on Personal, Indoor and Mobile Radio Communications (PIMRC'06)

TABLE II

REQUiRED NUMBERS OF REAL MULTIPLICATIONS IN MLD, QRM-MLD, AND MULTI-QRD-GD.

\begin{tabular}{l||l|l|l}
\hline Operation & MLD & QRM-MLD & Multi-QRD-GD \\
\hline \hline QR-decompositions & - & $4 L^{3} K$ & $8 L^{3} K$ \\
\hline Computation for $\boldsymbol{Q}^{H}(t, k) \boldsymbol{r}(t, k)$ & - & $4 L^{2} K D$ & $4 L^{2} K D$ \\
\hline Replica symbols generation & $4 L^{2} S K$ & $2 L(L+1) S K$ & $L(L+2) S K$ \\
\hline Computation for metrics & $2 L S^{L} K D$ & $2\left(1+\sum_{l=1}^{L-1} S_{l}\right) S K D$ & $4\left(1+S_{1}\right) S K D$ \\
\hline \hline Total (QPSK) & 30081024 & $\begin{array}{l}2770944 \\
\left(\left[S_{1}, S_{2}, S_{3}\right]=[4,4,4]\right)\end{array}$ & $2568192\left(S_{1}=4\right)$ \\
\hline Total (16QAM) & 7651196928 & $\begin{array}{l}18898944 \\
\left(\left[S_{1}, S_{2}, S_{3}\right]=[12,12,12]\right)\end{array}$ & $17498112\left(S_{1}=16\right)$ \\
& & $15630336\left(S_{1}=14\right)$ \\
\hline
\end{tabular}

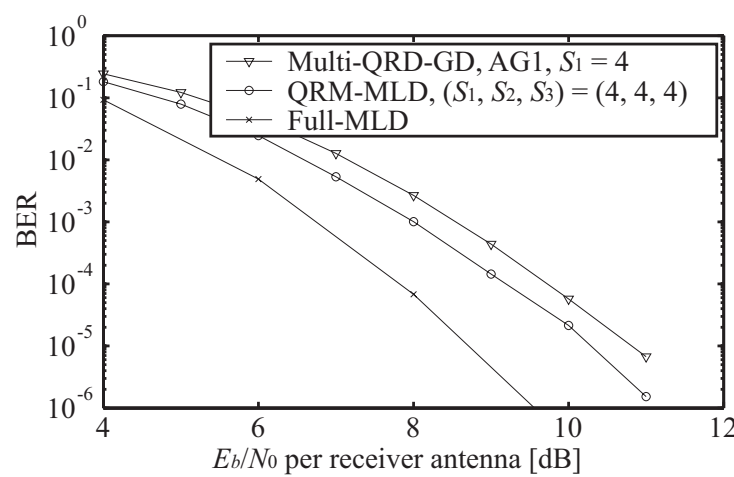

(a) QPSK

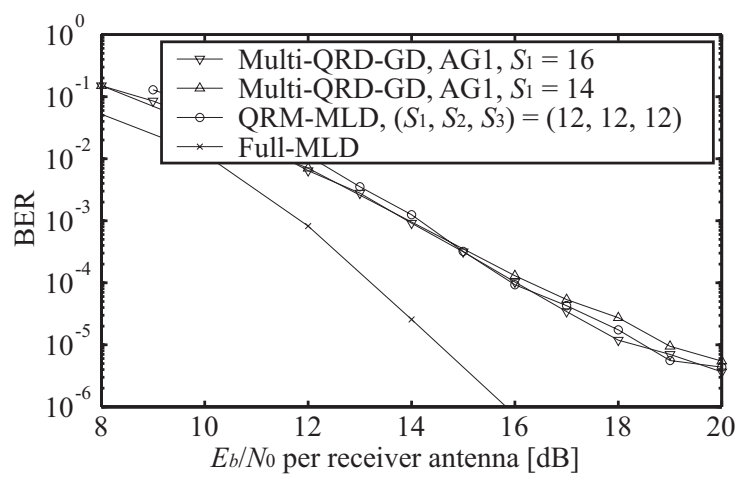

(b) 16QAM

Fig. 6. BER comparison of Full-MLD, QRM-MLD, and Multi-QRD-GD.

\section{CONCLUSION}

In this paper, we proposed a Multi-QRD-GD algorithm and AG scheme for the development of reduced-complexity-and-latency MIMO-OFDM receivers. Multi-QRD-GD has an equivalent BER performance to the conventional detection algorithms, despite it requires less computational complexity and latency in the signal processing than the conventional ones. In our future work, we must evaluate a MIMO-OFDM system including our proposed Multi-QRD-GD and SUCE in a fast time-varying fading environment. In addition, we must generalize our Multi-QRD-GD algorithm without any dependence to the number of the multiple antennas.

\section{ACKNOWLEDGEMENTS}

This work is partly supported by Research Fellowships of the Japan Society for the Promotion of Science for Young Scientists.

\section{REFERENCES}

[1] G.D. Golden, C.J. Foschini, R.A. Valenzuela, and P.W. Wolniansky, "Detection algorithm and initial laboratory results using V-BLAST space-time communication architecture," Electron. Lett., vol.35, no.1, pp.14-16, Jan. 1999.

[2] R. van Nee, and R. Prasad, OFDM for Wireless Multimedia Communications, Artech House, 1999.

[3] A. van Zelst, and T.C.W. Schenk, "Implementation of a MIMO OFDMbased wireless LAN system," IEEE Trans. Signal Processing, vol.52, no.2, pp.483-494, Feb. 2004.

[4] J. Penketh, and M. Collados, "Performance and implementation complexity of receiver algorithms for MIMO-OFDM based wireless LAN systems,” Proc. of IEEE PIMRC 2004, vol.2, pp.1522-1526, Sept. 2004.

[5] A. van Zelst, R. van Nee, and G.A. Awater, "Space division multiplexing (SDM) for OFDM systems," Proc. of IEEE VTC 2000-Spring, vol.2, pp.1070-1074, May 2000.

[6] V. Pammer, Y. Delignon, W. Sawaya, and D. Boulinguez, "A low complexity suboptimal MIMO receiver: the combined ZF-MLD algorithm," Proc. of IEEE PIMRC 2003, vol.3, pp.2271-2275, Sept. 2003.

[7] E. Viterbo, and J. Boutros, "A universal lattice code decoder for fading channels,” IEEE Trans. Information Theory, vol.45, no.5, pp.1639-1642, July 1999.

[8] T. Cui, and C. Tellambura, "Approximate ML detection for MIMO systems using multistage sphere decoding," IEEE Signal Processing Lett., vol.12, no.3, pp.222-225, March 2005.

[9] X. Li, H.C. Huang, A. Lozano, and G.J. Foschini, "Reduced-complexity detection algorithms for systems using multi-element arrays," Proc. of IEEE GLOBECOM '00, vol.2, pp.1072-1076, Dec. 2000.

[10] T. Aoki, H. Matsuoka, and H. Shoki, "Novel reduced complexity algorithm based on group detection in multiple antenna systems," Proc. of IEEE PIMRC 2004, vol.2, pp.1444-1448, Sept. 2004.

[11] G.A. Awater, A. van Zelst, and R. van Nee, "Reduced complexity space division multiplexing receivers," Proc. of IEEE VTC 2000-Spring, vol.1, pp.11-15, Sept. 2000.

[12] K.J. Kim, and J. Yue, "Joint channel estimation and data detectin algorithms for MIMO-OFDM systems," Thirty-Sixth Asilomar Conference on Signals, Systems and Computers, pp.1857-1861, Nov. 2002.

[13] H. Kawai, K. Higuchi, N. Maeda, M. Sawahashi, T. Ito, Y. Kakura, A. Ushirokawa, and H. Seki, "Likelihood function for QRM-MLD suitable for soft-decision turbo decoding and its performance for OFCDM MIMO multiplexing in multipath fading channel," IEICE Trans. Commun., vol.E88-B, no.1, pp.47-57, Jan. 2005.

[14] R. Funada, H. Harada, and S. Shinoda, "Performance improvement of decision-directed OFDM channel estimation in a fast fading environment," IEICE Trans. Fundamentals, vol.E87-A, no.8, pp.1994-2001, Aug. 2004

[15] K. Yokomakura, S. Sampei, H. Harada, and N. Morinaga, "A carrier interferometry based channel estimation technique for one-cell reuse MIMO-OFDM/TDMA cellular systems," IEEE VTC 2006-Spring, Melbourne, Australia, 7-10 May 2006. 